\title{
A Systematic Approach to Predict the Behavior of Cough Droplets Using Feedforward Neural Networks Method
}

\author{
Irfan Bahiuddin ${ }^{1, *}$, Setyawan Bekti Wibowo ${ }^{1}$, M. Syairaji ${ }^{2}$, Jimmy Trio Putra ${ }^{3}$, Cahyo Adi Pandito ${ }^{1}$, \\ Ahdiar Fikri Maulana ${ }^{4}$, Rian Mantasa Salve Prastica ${ }^{5}$ and Nurhazimah Nazmi ${ }^{6}$ (D) \\ 1 Department of Mechanical Engineering, Vocational College, Universitas Gadjah Mada, \\ Yogyakarta 55281, Indonesia; setyawanbw@ugm.ac.id (S.B.W.); panditodraci@gmail.com (C.A.P.) \\ 2 Department of Information and Medical Service, Vocational College, Universitas Gadjah Mada, \\ Yogyakarta 55281, Indonesia; msyairaji@ugm.ac.id \\ 3 Department of Electrical Engineering and Informatics, Vocational College, Universitas Gadjah Mada, \\ Yogyakarta 55281, Indonesia; jimmytrioputra@ugm.ac.id \\ 4 Department of Bioresources Technology and Veterinary, Vocational College, Universitas Gadjah Mada, \\ Yogyakarta 55281, Indonesia; ahdiar.f.maulana@ugm.ac.id \\ 5 Department of Civil Engineering, Vocational College, Universitas Gadjah Mada, Yogyakarta 55281, Indonesia; \\ rian.mantasa.s.p@ugm.ac.id \\ 6 Malaysia-Japan International Institute of Technology, Universiti Teknologi Malaysia, Jalan Sultan Yahya Petra, \\ Kuala Lumpur 54100, Malaysia; nurhazimah@utm.my \\ * Correspondence: irfan.bahiuddin@ugm.ac.id
}

Citation: Bahiuddin, I.; Wibowo, S.B.; Syairaji, M.; Putra, J.T.; Pandito, C.A.; Maulana, A.F.; Prastica, R.M.S.; Nazmi, N. A Systematic Approach to Predict the Behavior of Cough Droplets Using Feedforward Neural Networks Method. Fluids 2021, 6, 76. https://doi.org/10.3390/fluids6020076

Academic Editor: Toni Lassila Received: 30 December 2020

Accepted: 1 February 2021

Published: 9 February 2021

Publisher's Note: MDPI stays neutral with regard to jurisdictional claims in published maps and institutional affiliations.

Copyright: (c) 2021 by the authors. Licensee MDPI, Basel, Switzerland. This article is an open access article distributed under the terms and conditions of the Creative Commons Attribution (CC BY) license (https:// creativecommons.org/licenses/by/ $4.0 /)$.
Abstract: Coronavirus disease 2019 (Covid-19) has been identified as being transmitted among humans with droplets from breath, cough, and sneezes. Understanding the droplets' behavior can be critical information to avoid disease transmission, especially while designing a device deals with human air respiratory. Although various studies have provided enormous computational fluid simulations, most cases are too specific and quite challenging to combine with other similar studies directly. Therefore, this paper proposes a systematic approach to predict the droplet behavior for coughing cases using machine learning. The approach consists of three models, which are droplet generator, mask model, and free droplet model modeled using feedforward neural network (FFNN). The evaluation has shown that the three FFNNs models' accuracies are relatively high, with R-values of more than 0.990 . The model has successfully predicted the evaporation effect on the diameter reduction and the completely evaporated state, which can be considered unlearned cases for machine learning models. The predicted horizontal distance pattern also agrees with the data in the literature. In summary, the proposed approach has demonstrated the capability to predict the diameter pattern according to the experimental or previous work data at various mask face types.

Keywords: droplet; cough; feedforward neural network; machine learning; respiratory system; empirical model

\section{Introduction}

The current issue of the COVID-19 pandemic has gained enormous interest to prevent virus transmission. Further understanding of the fluids and particle transport from the respiratory behavior is vital to building countermeasures against the disease. The transport can be studied based on the dynamics of the respiratory droplets. The investigation of droplet behavior can be either computational fluid dynamics or experimental methods. The droplet characteristic can be described in terms of the diameters, temperature, droplet diameter distribution, number of droplets, evaporation times, falling time to the ground, density, contaminant, and others [1-3].

Various studies have tried to discover the droplet dynamic related-transport mechanism, such as the droplet generation's factors and how the droplet dynamics after coming out of the mouth. The human as the droplet source is one of the main factors affecting the 
droplet group characteristics, including distribution, mass flow, and droplet number [1]. Human activity, including breathing [4-7], coughing [1,8-11], sneezing [12], mouth open or not [1], is one of the obvious factors affecting the droplet group characteristics. Mask efficiency can also affect droplet transportation [2,3] depending on the materials or the filtration capability. According to the experiment by Verma et al. [3], the mask can affect the flow jet direction, the velocity outflow, and the released droplets. While those factors affect the initial droplet groups' characteristics, the environment can affect the droplet dynamics after coming out of the mouth, including the horizontal distance penetration, evaporation rate, and the falling time $[10,13]$. The previous investigation's usual environment variables are humidity, wind velocity, temperature, and ventilation [13-16].

Many simulation and experimental works have tried to discover the mechanism behind the droplet behavior at various conditions. The correlation between the affecting factors and the droplet group properties is usually discovered or calculated using the numerical method [17-21]. Each work is usually carried out for simulating a specific case. As widely known, numerical simulations are powerful methods to discover a complex system mechanism. The computational burden depends on simulation complexity. There is a high number of experimental data and simulation results available in literature after going through arduous effort and time-consuming processes. However, if new variables are needed to be considered, more time-consuming simulations need to be carried out. Empirical models should be potential methods to gain more benefit by predicting the droplet behavior straightforwardly based on the available data based on the previous research. In other words, an approach that is possibly capable of accommodating all the available measured data and simulation results is needed, especially to apply in practical cases or fast prediction of the droplet behavior. For example, the model can be employed to directly predict droplet behavior based on the database in literature without re-run a complex simulation using a numerical method.

However, the effort to develop empirical models that can flexibly accommodate various variables and correlation can be considered rare. One of the works [22] have carried out experiments about the flow rate, flow direction, and air velocity determined from the flow rate and mouth opening area and develop an empirical model. The model inputs are the height, weight, and gender of a person. The model focuses more on the human factor, including age, gender, and height, with the produced droplets. Another simplified model related to the respiratory droplet is in [23] using the Maxey Riley equation that has successfully analyzed droplet behavior, especially in horizontal penetration. This model has limitations only for a specific case hence limited variables coverage.

Machine learning is one possible solution that can accommodate various empirical data and numerical simulation results to produce more meaningful insight. Machine learning, including extreme learning machine (ELM), artificial neural networks (ANN) have gained enormous attention because its capability to predict with high accuracy and recognize the pattern of a complex system. Machine learning consists of training algorithms and topology. Algorithm is a method to training the topology, such as backpropagation (BP), ELM, and deep learning (DL). The topology is about the model structure that will be trained using the selected algorithm. Feedforward neural network (FFNN) is the most popular topology employed in various cases and can model almost all complex cases [24,25].

Therefore, this paper proposes a novel framework to predict droplet behavior using a machine learning method based on empirical data and numerical results. The proposed model is developed based on FFNN built by an extreme learning machine (ELM) algorithm. The current work is limited to only cough cases and zero wind conditions, with the assumption of a free fall droplet. Firstly, the methodology is proposed, including the proposed method's general concept, the model developed for each part of the proposed method, and the respective parameter set up. Then, the method's capability to predict droplet behavior is evaluated and discussed. 


\section{Methodology}

\subsection{Proposed Method}

Figure 1 shows the three main parts of the proposed approach, which are the droplet generator, mask models, and droplet behavior in the environment. The droplet generator consists of algorithms to generate a set of data representing the droplet. Mask models consist of the efficiency of the prediction and to simulate the trapping process of the droplets. The part of the droplets in the environment means to predict horizontal distance and evaporation rates.

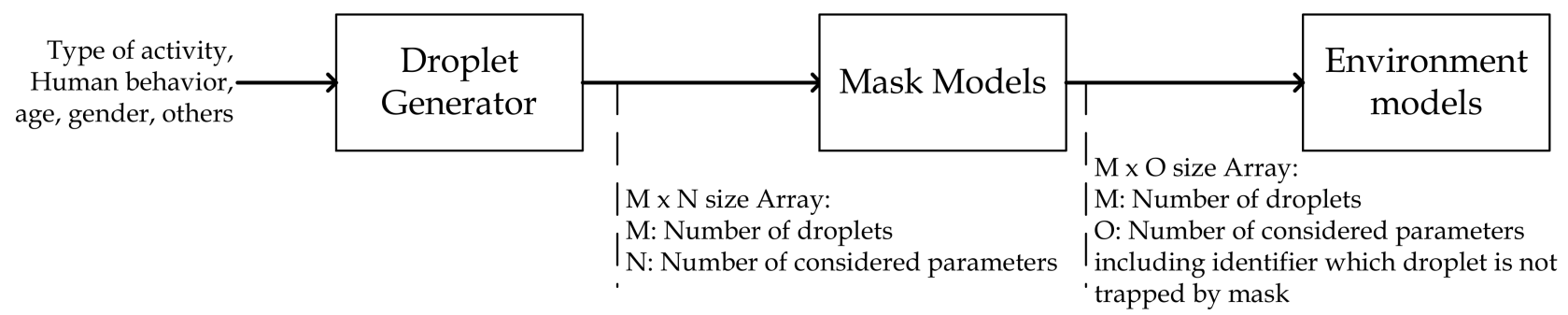

Figure 1. The Proposed Systematic Approach to predict Droplet Distributions.

The machine learning algorithm employed to build FFNN is Extreme learning machine (ELM). ELM has known for its fast training time and comparable accuracy compared to other popular methods, such as support vector regression (SVR) and backpropagation (BP) [26,27]. ELM builds a model without iteration by assigning random values to the weighting of input and then calculating the output weighting by applying pseudoinverse [28]. The data for modeling is divided into only two groups, which are training and testing. While the training data is for building the model, the testing data is a group of distinct data to prove that the model can work well. The model in the training process should not learn testing data. All data in this manuscript is divided into $80 \%$ of training and $20 \%$ of testing data. The normalization is applied by employing min-max linear normalization.

\subsection{Droplet Generator}

The mean flow rate for coughing for the males between $20-30$ is $0.48 \mathrm{~L} / \mathrm{s}$, with standard deviations is $0.09 \mathrm{~m} / \mathrm{s}$. The current work focuses on the model formulated based on the measured droplet while coughing with mouth open. The data is shown in Figure 2 from [1] The droplet is then generated based on the designated range according to the data. The further variation will be developed in the future to cover broader human factors and more activities. The data can be found in various literature, such as gender, height, weight [13], and age [11]. The activity can also affect the diameter distribution, such as coughing [1,11], breathing $[1,4]$, and sneezing [1,12]. The matrix of the generated droplet can be represented in Equation (1), where $M$ is the number of droplets, from the diameter value for the first droplet $d_{1}$ to the $M$-th droplet $d_{M}$. According to the literature [1], the number of droplet is 3000 .

$$
D=\left[d_{1}, d_{2}, d_{3}, \ldots, d_{M}\right]^{\mathbf{T}}
$$




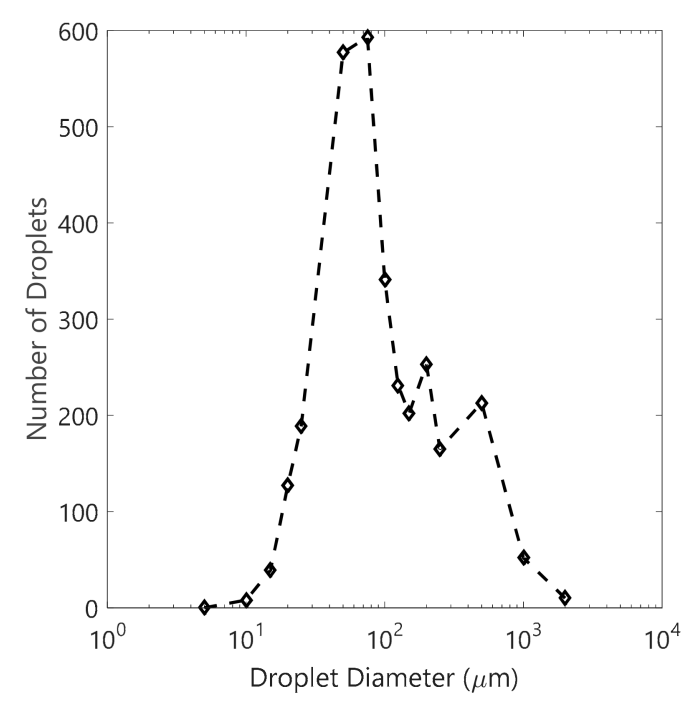

Figure 2. The droplet distribution according to the data in [1].

\subsection{Mask Model}

The masked model is built using an FFNN method based on the data in [29] to predict the efficiency. The employed model has two parts, which are the efficiency model and determination of the trapped droplet. The efficiency model is built based on FFNN inspired by the biological nervous system. The FFNN model is known to replicate a system behavior, especially highly nonlinear models. A critical step of the FFNN model development is the determination of input and output. For the efficiency model, the inputs are diameter $d$ and type of the mask $(k)$, and the output is the efficiency $\eta$ represented by the function shown in Equation (2)

$$
\eta=f(d, k)
$$

The function is an FFNN model with one input, one hidden node, and one output layer. The function is also called a multi-layer perceptron with the three layers, as mentioned before. The training model is trained using an extreme learning machine (ELM) to predict with acceptable generalization $[24,25]$. Another method can also be employed to train the FFNN model, such as Levenberg Marquardt [30].

The training data is a set of mask efficiency as a function of diameter at various mask types from [29], as shown in Figure 3. The accommodated mask types are cotton, gauze, N95, procedure, and surgical masks. The numbering is started from 0 representing no mask condition to 5 representing the N95 mask. The data is divided into two groups, which are training consisting of $80 \%$ data and testing consisting of $20 \%$ data. The training data is employed for developing the model. Testing data is utilized to predict the obtained model performance or outside of the training process.

As described in the earlier paragraph, the training method is an ELM [31] using the basic method. The employed activation function is a hard limit, as it has shown a good agreement in various cases [32,33]. The hidden node number is varied from 10 to 10,000 , as the model has shown an acceptable computational time and agreeable accuracy for other cases $[31,33]$.

The determination of whether the mask traps a specific droplet or not is by comparing the efficiency value of a randomly generated using a uniform distributed function. If the random value is less than the predicted efficiency, the coupled droplet is classified as trapped by the masker. Otherwise, the droplet is then passed to the next stage. With an $\boldsymbol{M}$ sample or droplet number, a set of numbers with the size of $M \times 1$ is generated using a uniformly distributed random function with a built-in-algorithm of Matlab. The array is then coupled with the obtained efficiency, and the value is compared with the predicted efficiency. If the droplet diameter has a diameter more than the training data's maximum 
value, the droplet is considered trapped except for the no mask condition. This model can be further developed in the future to cover the velocity direction and magnitude, and mass flow of the droplet according to the experimental data [3] or simulation data.

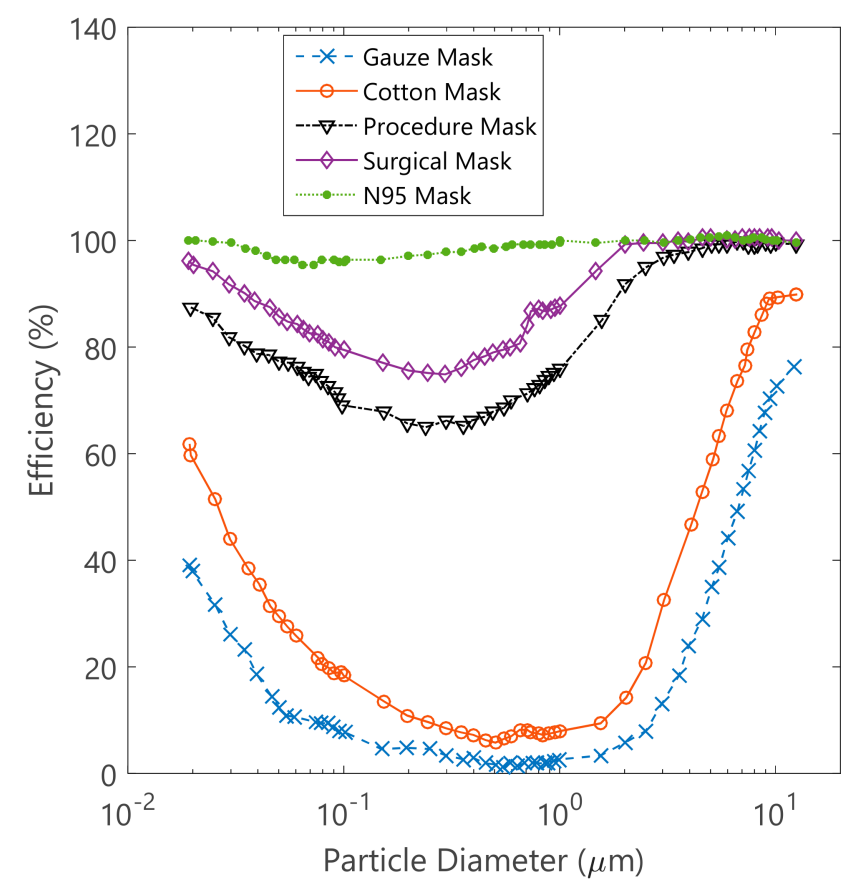

Figure 3. Efficiency data of the mask are taken from [29].

The mask model limitation depends on the available data related to mask efficiency, which is about $12 \mu \mathrm{m}$. If the droplet diameter is more than $12 \mu \mathrm{m}$, the maximum efficiency $\eta_{\text {high }}$ according to the mask type (see Table 1 ) is applied. The pseudo-code for the determination of the efficiency is shown below.

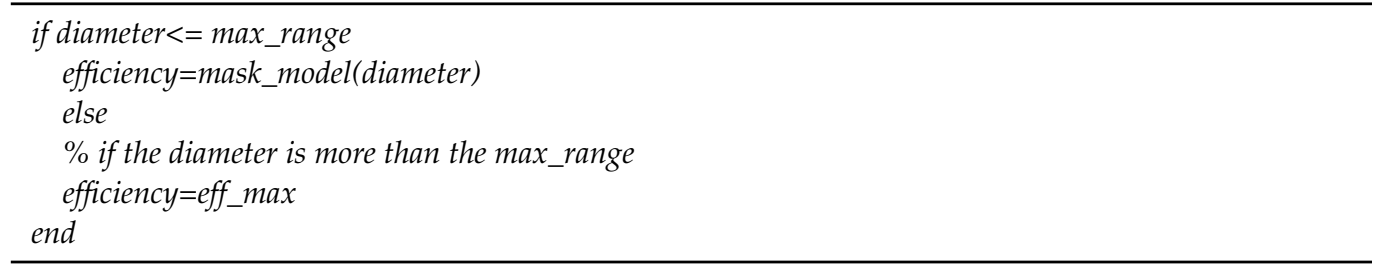

Table 1. The highest and lowest efficiency of face mask ( $\eta_{h i g h}$ and $\eta_{\text {Low }}$, respectively).

\begin{tabular}{ccc}
\hline Mask & $\eta_{\text {Low }}(\mathbf{\%})$ & $\eta_{\text {high }}(\mathbf{\%})$ \\
\hline No Mask & 0 & 0 \\
Gauze & 1 & 77.0 \\
Cotton & 6 & 90.0 \\
Procedure & 65 & 99.7 \\
Surgical & 75 & 100.0 \\
N95 & 95 & 100.0 \\
\hline
\end{tabular}

If the first part of the model generates the model ranging from $1 \mu \mathrm{m}$ to $2 \mathrm{~mm}$, the droplet with a diameter of more than $12 \mu \mathrm{m}$ is determined to be trapped or not based on the maximum efficiency $\eta_{\text {high }}$ in Table 1.

\subsection{Environment Models}

The droplets come along the respiratory jet and eventually leave the jet and dropped to the ground or evaporated, as shown in Figure 4. The total evaporation time and the falling 
time to the ground with a $2 \mathrm{~m}$ distance of the droplet can be depicted in Figure 5. The smaller droplet evaporates, and the giant droplet will drop to the ground. The evaporation rate, evaporation time, and the time to fall to the ground is a function of various variables, such as humidity and wind velocity [29]. Another important information is the penetration or the horizontal distance to know how far the diameter can travel that can be derived to consider safe distance in human to human interaction [22,34-37]. The formulation of the model is divided into two cases, which are the model to predict the droplet diameters reduction and the prediction of the maximum horizontal distance.

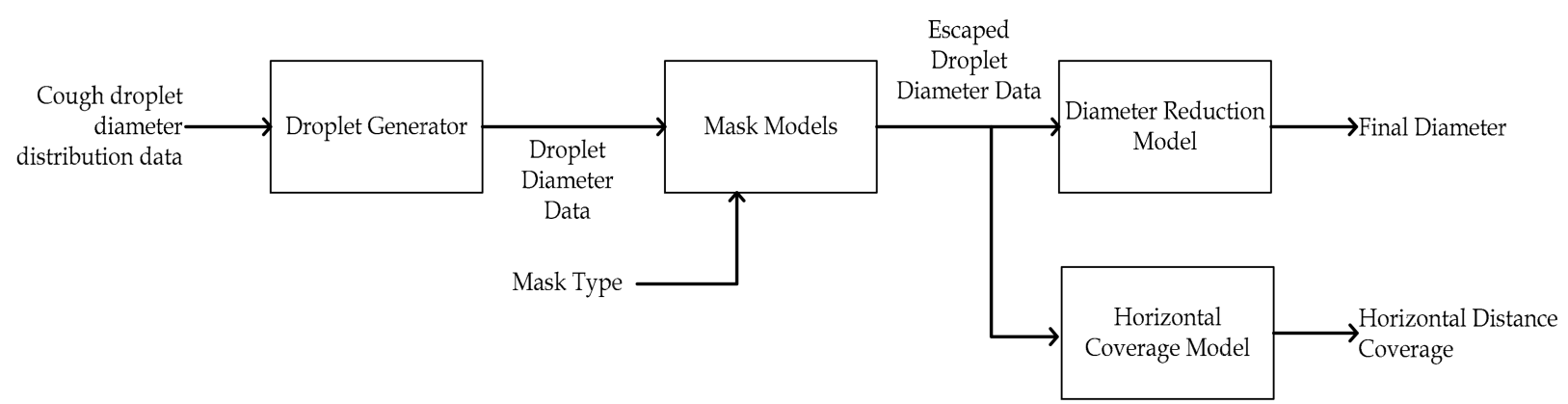

Figure 4. The environmental models for predicting the droplet diameter reduction and horizontal coverage.

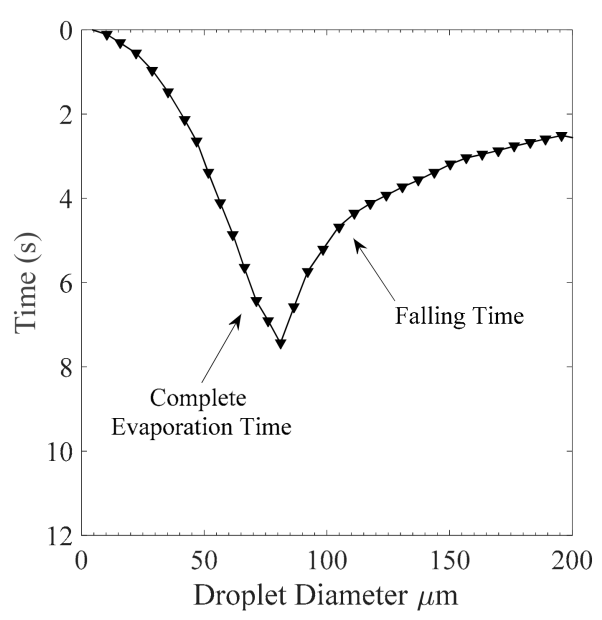

Figure 5. The evaporation and falling time for a droplet with temperature $33^{\circ} \mathrm{C}$ and $\mathrm{RH} 50 \%$ [37].

The first model is formulated to predict the diameter as a function of time and the droplet's initial size. Therefore a data based on numerical simulation in [37] is employed as the training data. The plot of the training data is depicted in Figure 6. The diameter is gradually decreased because the evaporation process becomes the airborne or droplet nuclei. The modeling method is an ELM (ELM) by employing an FFNN with a single hidden layer. The mathematical representation of the model is shown in Equation (3), where $t$ is the time after coming out of the mouth, $d_{0}$ and $d_{t}$ are the initial and later state of the droplet diameter, respectively. The FFNN employs the 500 hidden node number and sigmoid activation function.

$$
d_{t}=f\left(t, d_{0}\right)
$$

The second model is developed to predict penetration ability as a function of droplet diameter. An FFNN with ten hidden node numbers and sigmoid activation is employed. The FFNN is trained using the ELM method. The data for the training data is taken from [37]. 


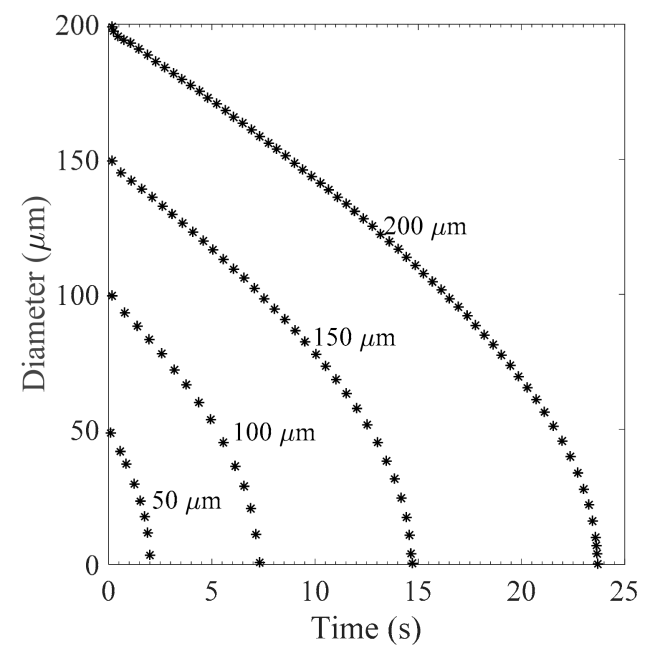

Figure 6. The Droplet Diameter Reduction as a function of time [37].

\section{Results and Discussions}

\subsection{Mask Model Evaluation}

The discussion consists of the model accuracy performance and the physical meaning of the predicted droplets. The simulation is firstly carried out by comparing various FFNN configurations. The 4000 hidden is selected because it has better accuracy than the fewer hidden node number and almost the same in terms of root mean square error (RMSE) with the higher hidden node number. The computational time is also quite acceptable, with training time less than $1 \mathrm{~s}$ while being simulated in Matlab installed in a computer with AMD processor Ryzen 3 and RAM 8 GB. After running the simulation, the results has shown a good agreement with the RMSE value of 0.0022 for the training case and 0.0042 for testing. The prediction of the efficiency can be checked visually in Figure 7 . The $R^{2}$ of the training and testing data of the mask, the model is more than 0.997 and 0.989 , respectively, which is considered high for correlation, as shown in Table 2.

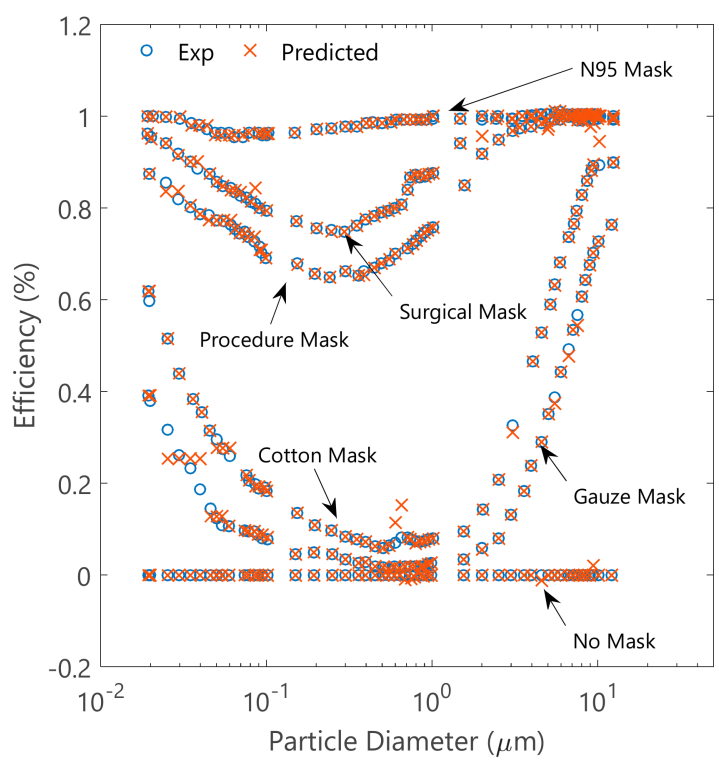

(a)

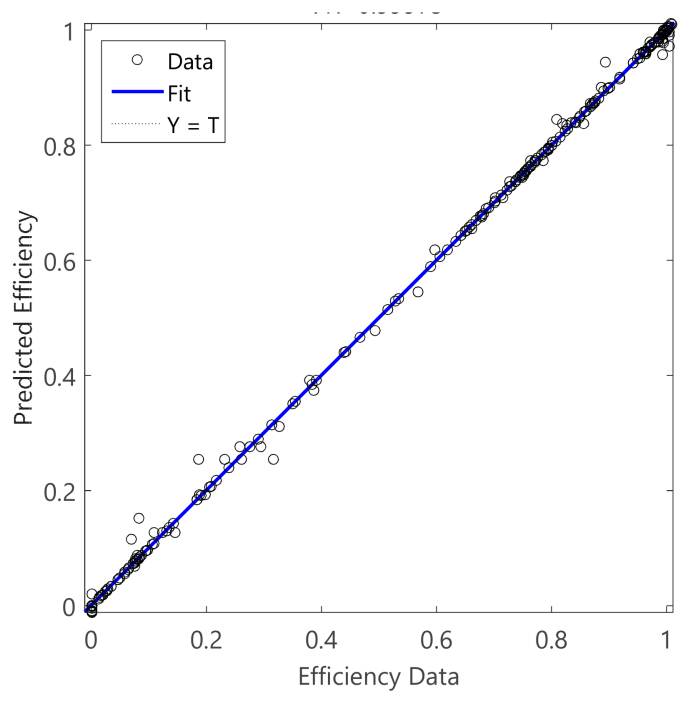

(b)

Figure 7. Comparison between the training data [29] and simulation results (a), the training correlation (b). 
Table 2. The accuracies of the proposed methods.

\begin{tabular}{cccc}
\hline Models & Data & RMSE & $\mathbf{R}^{\mathbf{2}}$ \\
\hline \multirow{2}{*}{ Mask model } & Training Data & 0.022 & 0.997 \\
& Testing Data & 0.042 & 0.989 \\
\hline \multirow{2}{*}{ Diameter Reduction model } & Training Data & 13.378 & 0.999 \\
& Testing Data & 26.798 & 0.994 \\
\hline \multirow{2}{*}{ Horizontal distance model } & Training Data & 0.039 & 0.990 \\
& Testing Data & 0.057 & 0.933 \\
\hline
\end{tabular}

Table 3 describes the droplet number after coming out of the mask, the percentage of the outlet volume compared to the initial volume, and the escaped droplet's diameters average. N95 has the highest capability as it traps the most number of droplets compared to others, with gauze as the least. The data is highly dependent on the training data. If the droplet diameter more than the maximum value of the training data, the efficiency values in Table 1 are employed depending on the mask type. The droplet number comparison of the procedure, surgical and N95 mask is almost the same. In reality, the N95 mask should have more efficiency compared to the surgical mask. The cause can be the reference for the cough droplet data distribution [1], where the small diameter droplet number with a size of less than $10 \mu \mathrm{m}$ is quite few. An addition simulation is added to check the mask accuracy further by adding the droplet number up to 100 for the range between 0 and $10 \mu \mathrm{m}$, the comparison is shown in the form of box plot for 50 times simulation as shown in Figure 8. The difference between N95 and surgical mask becomes more apparent. From the results, it can be said that the proposed mask model can predict the escaped droplets. The comparison between the surgical and N95 mask can be further compared in the future, therefore it can be almost the same as in [3], especially in terms of the trapped droplet number. In the future, the mask training data will be updated according to the latest and more accurate sources.

Table 3. The escaped droplet of the mask (depending on the training data pattern).

\begin{tabular}{cccc}
\hline Condition & Droplet Number & The Outlet Vol (\%) & Average Diameter $(\mu \mathbf{m})$ \\
\hline No Mask & 3000 & 100.0 & 121.1 \\
Gauze & 695 & 15.7 & 111.6 \\
Cotton & 309 & 17.4 & 121.7 \\
Procedure & 7 & $5.0 \times 10^{-3}$ & 58.3 \\
Surgical & 1 & $1.1 \times 10^{-12}$ & 0.1 \\
N95 & 0 & 0 & 0.0 \\
\hline
\end{tabular}

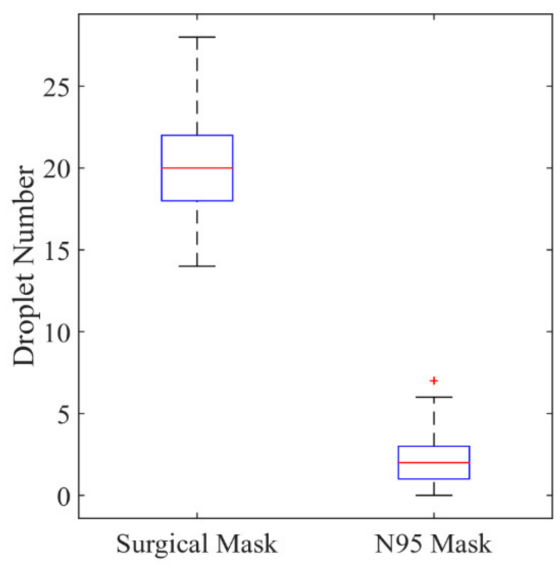

Figure 8. Box plot comparison of a surgical mask and N95 mask after 50 times running at additional droplet generator setting. 


\subsection{The Droplet Evaporation and Penetration}

As discussed in the methodology, the model consists of two parts, the reduced diameter of the droplet and the penetration capability. Both models were modeled using FFNN trained by ELM algorithms. The predicted reduced diameters were compared with the data from [37] or data in Figure 5. As shown in Figure 9, the predicted data has shown a good agreement with the training data. Although some slight errors have been found in the small value of diameter data, the overall regression analysis between the predicted droplet diameter and the training and testing data's diameter has shown a high correlation with 0.999 and 0.994 of $R^{2}$, respectively. The model represented by Figure 9 is to predict the droplet diameter reduction behavior after escaping from mouth or mask. Figure $9 b, c$ shows the comparison of the predicted diameter at a certain timestamp. The model range is actually up to $1500 \mu \mathrm{m}$ to accommodate the no mask condition. Furthermore, for the droplets with diameter less than $10 \mu \mathrm{m}$, the evaporation process will be very fast about less than $1 \mathrm{~s}$ and will not move too faraway from the initial position (except there is wind from external environment). Therefore, while observing the horizontal distance, the error can be ignored. However, to reduce the indicated error of the small particles in Figure 9c, the proposed methods will be refined in the future by accommodating more comprehensive training data or by applying another machine learning method.

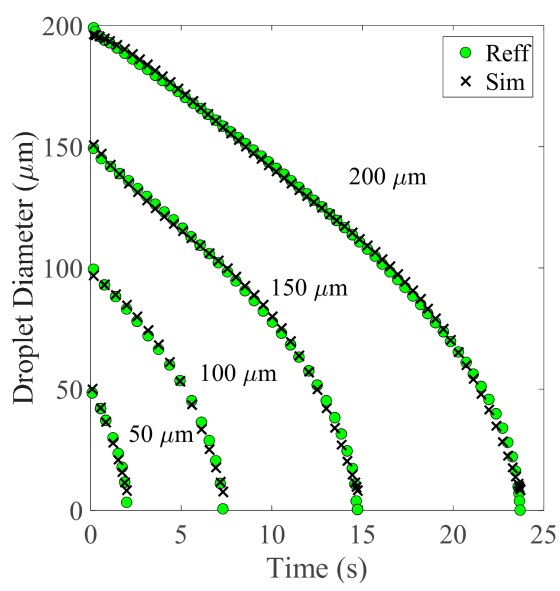

(a)

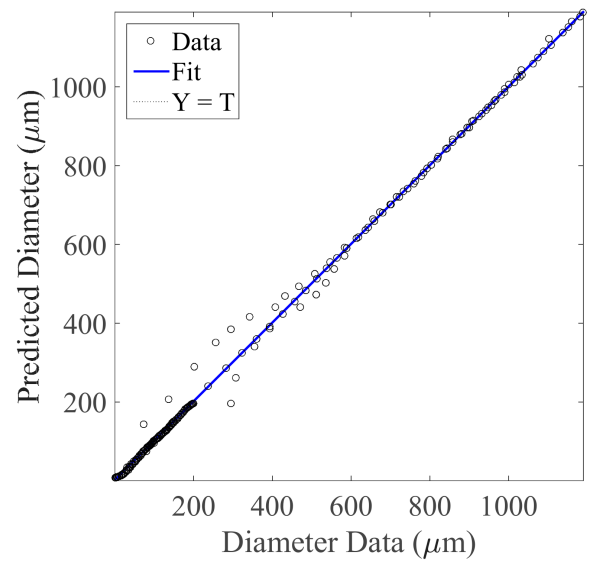

(b)

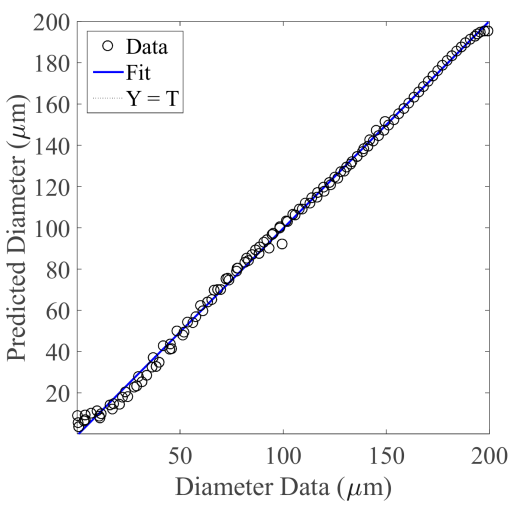

(c)

Figure 9. The droplet diameter reduction prediction compared to the reference data (a) as a function of time series, (b) the correlation between the predicted and reference data for all diameter data, and (c) for data with initial diameter less than $200 \mu \mathrm{m}$. 
The first and second data of Figure 10 shows reduced diameter and time as a function of the diameters. The $y$-axis is the predicted diameter at a specific timestamp from the first figure's FFNN model. As described in Section 2.3, the model's input is the time stamp and the initial diameter. The second figure is the input to obtain the $y$-axis of the first figure. The input data from [36] represents the falling time and evaporation time for various diameters. In other words, there are two regions. The first figure shows that the boundary's right side has a predicted diameter with negative values. In other words, the volume droplet is zero, or it has evaporated completely. Figure 10 also confirms that the model has shown its capability to predict the droplet behavior outside of the training data range or unlearned data. The training data only consist of the reduced diameter up to the evaporation time. In contrast, the data for what happens after the designated time is unknown from the model point of view. After running the simulation, the result has shown the negative value that can be interpreted to be evaporated completely. In summary, the model's capability to predict the evaporation condition and the final state of the diameter after a specific timestamp has been demonstrated.

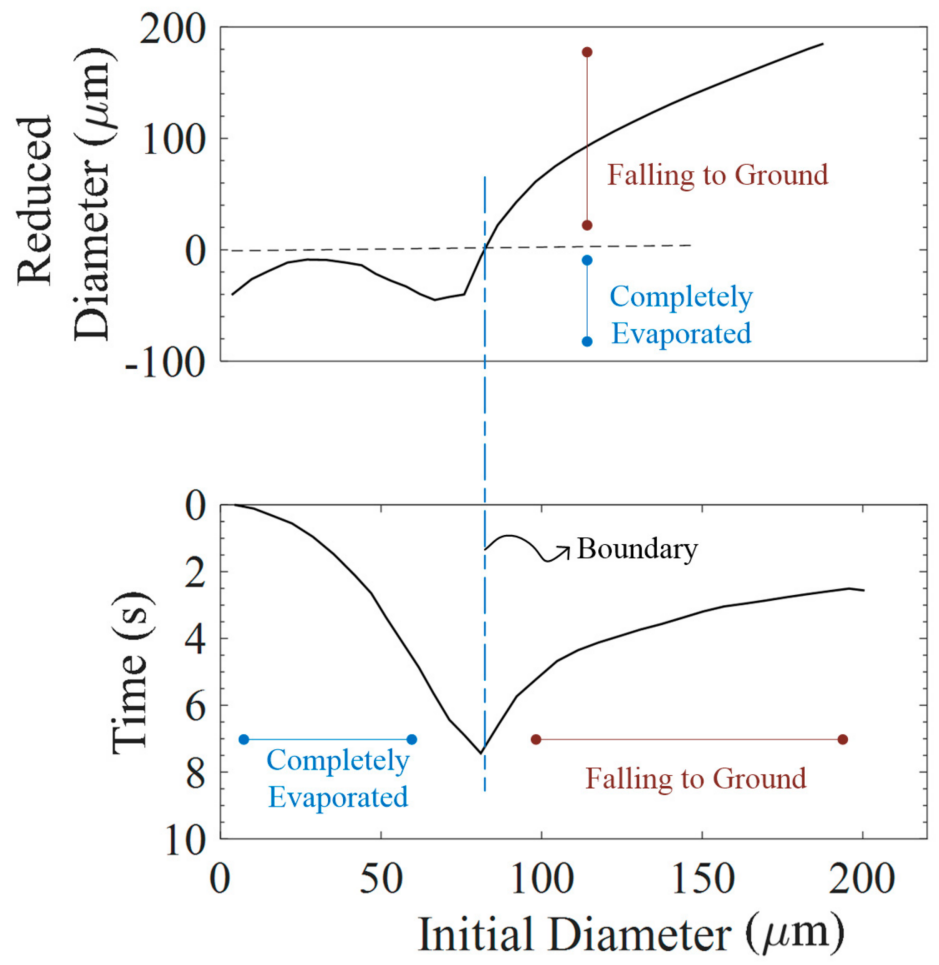

Figure 10. The region for the completely evaporated droplets and falling to the ground droplet after falling from $2 \mathrm{~m}$ height.

Table 4 shows the evaporated droplets at specific timestamps, which are 2 and $10 \mathrm{~s}$. Besides N95 with no escaped droplet, the droplets from other mask types have shown a considerable number of the evaporation process after a duration of $2 \mathrm{~s}$ flowing to the ambient air. After $10 \mathrm{~s}$, most of the droplets have evaporated while the diameter of the rest continuously decreases. For example, the remaining droplet number of the cotton mask is about 94, with the average diameter is $197.56 \mu \mathrm{m}$ which should be less than the initial diameter. Furthermore, the other remaining droplets would be evaporated completely or falling to the ground after a certain period, which one comes first. The analysis of the obtain data from this part of the model can be combined with the data from the next model that predicts horizontal distance coverage. 
Table 4. Diameter reduction for determining evaporation state at a specific timestamp.

\begin{tabular}{ccccc}
\hline \multirow{2}{*}{ Mask Type } & \multicolumn{2}{c}{ Evaporated Droplets } & \multicolumn{2}{c}{ Not Evaporated Droplets } \\
\cline { 2 - 5 } & $n_{e, 2 s}$ & $n_{e, 10 s}$ & $n_{f, 10 s}$ & $\boldsymbol{d}(\boldsymbol{\mu m})$ \\
\hline No Mask & 711 & 2020 & 980 & 171.26 \\
Gauze & 166 & 500 & 195 & 165.77 \\
Cotton & 82 & 215 & 94 & 197.56 \\
Procedure & 2 & 6 & 1 & 67.89 \\
Surgical Mask & 1 & 1 & 0 & 0.00 \\
N95 & 0 & 0 & 0 & 0.00 \\
\hline
\end{tabular}

$n_{e, 2 s}$ : evaporated droplet number at $2 \mathrm{~s}$ after coming out of the mouth, $n_{e, 10 s}$ : evaporated droplet number at $10 \mathrm{~s}$ after coming out of the mouth/face mask, $n_{f, 10 s}$ : non-evaporated droplet number at $10 \mathrm{~s}$ after coming out the mouth/face mask, $\mathrm{d}$ : the average of the droplet number.

The horizontal distance predictor or the second model has been compared with the training data from [37], as depicted in Figure 11a as the function of diameter and b regression analysis. The model has a relatively high correlation value for training and testing, which are 0.990 and 0.933 , respectively, as shown in Table 2 . The model also can follow the training data pattern by showing the furthest distance of the droplet is covered by droplets with a diameter of about $30 \mu \mathrm{m}$. If the input droplet diameter value is more than the training data's coverage, the highest value of diameter input is considered, which is the same as in the mask model. The horizontal distance coverage is affected by various variables, such as humidity, the initial velocity of the droplet, wind velocity from ambient or ventilation that can be considered in the future.

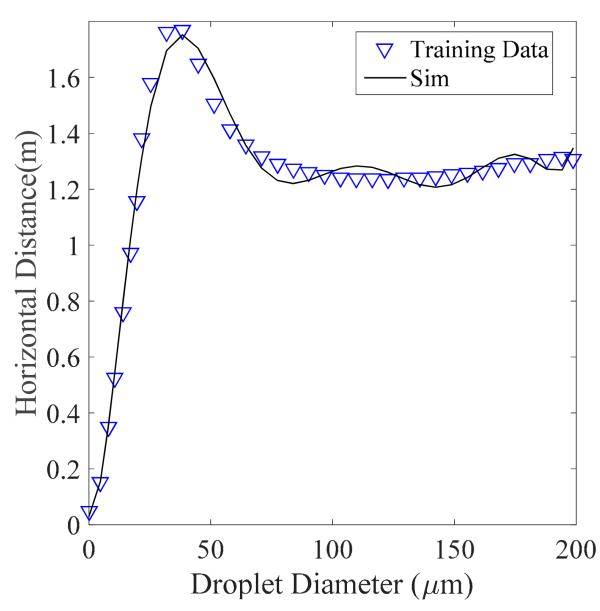

(a)

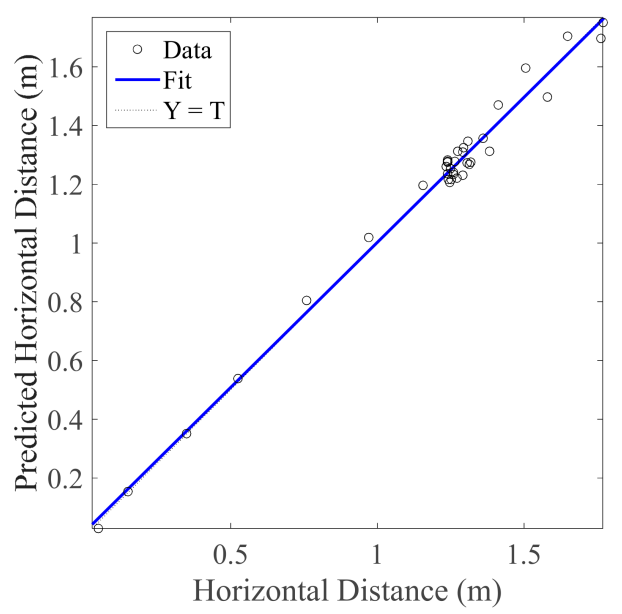

(b)

Figure 11. The horizontal distance prediction compared to the numerical model as a function of droplet diameter (a) and the training correlation (b).

Table 5 shows the predicted droplet number at various ranges of horizontal distance. N95 and surgical mask show zero value of droplets because the escaped droplets have a relatively small size and will be evaporated at a relatively short distance. The highest droplet number is found at no mask condition, followed by gauze, cotton, and procedure. The droplet number at a horizontal distance of less than $50 \mathrm{~cm}$ is not shown in the model because the number is only minority and the droplets will be completely evaporated because of the small diameter. From the table, N95 and the surgical mask has the safest condition because of the minimum values of droplet number at a distance of more than $50 \mathrm{~cm}$. From the droplet generators' point of view, if the droplet number for small size diameter is added in the cough droplet distribution, the higher number escaped droplets from the mask will be likely evaporated before reaching a distance of more than $50 \mathrm{~cm}$ because of the small size of the droplet. For the procedure mask, 5 droplets are detected at 
a distance of more than $100 \mathrm{~cm}$, significantly lower than cotton and gauze masks. When considering the no-mask condition, the droplet number majority is found between 100 and $140 \mathrm{~cm}$. The droplet can be 3 times lower than the no mask condition by putting the gauze mask on. The droplet population can be further reduced by half of the original by putting on the cotton mask. In other words, although the gauze mask has significantly low effectiveness compared to the surgical mask and N95 mask, the gauze mask still can considerably reduce the droplet volume three times than the no mask condition.

Table 5. The predicted droplet number at various horizontal distance ranges.

\begin{tabular}{|c|c|c|c|c|c|c|}
\hline \multirow{3}{*}{ Mask Type } & \multicolumn{2}{|c|}{$\left[x_{50}, x_{100}\right]$} & \multicolumn{2}{|c|}{$\left[x_{100}, x_{140}\right]$} & \multicolumn{2}{|c|}{$\left[x_{140}, x_{180}\right]$} \\
\hline & & - & & - & & - \\
\hline & $n$ & $d(\mu \mathrm{m})$ & $n$ & $d(\mu \mathrm{m})$ & $n$ & $d(\mu \mathrm{m})$ \\
\hline No Mask & 75 & 13.94 & 1979 & 163.07 & 933 & 42.35 \\
\hline Gauze & 24 & 14.02 & 447 & 151.70 & 218 & 43.13 \\
\hline Cotton & 10 & 14.99 & 202 & 165.48 & 93 & 43.25 \\
\hline Procedure & 0 & 0.00 & 2 & 79.88 & 3 & 42.15 \\
\hline Surgical Mask & 0 & 0.00 & 0 & 0.00 & 0 & 0.00 \\
\hline N95 & 0 & 0.00 & 0 & 0.00 & 0 & 0.00 \\
\hline
\end{tabular}

$\left[x_{50}, x_{100}\right]$ is horizontal direction between 50 and $100 \mathrm{~cm},\left[x_{100}, x_{140}\right]$ is between 100 and $140 \mathrm{~cm},\left[x_{140}, x_{180}\right]$ is between 140 and $180 \mathrm{~cm}, n$ is droplet number, $\bar{d}$ is the average diameter of the droplets at a designated distance range.

In summary, the proposed model has successfully demonstrated the capability to predict the droplet behavior at various mask types. The simulation time is also relatively fast. While the training time is less than one second, the prediction time can be faster. The duration to complete 50 times simulation involving 3000 droplets at 6 different mask conditions for the three models is about $312 \mathrm{~s}$ which is relatively fast. The manuscript's main novelty is about a framework to predict droplet behavior based on various literature's available data. Therefore, the method can be extended into some possible directions. Firstly, the model can be developed to accommodate the most updated training data due to the quality of a machine learning-based model depending on the quality of the data. Therefore, if a new finding appears in the future, the research can be accommodated by the model by including the training data's data. Secondly, the model can be developed further by accommodating more independent variables. For the droplet generator, another droplet distribution data at different human activities can be added in the future. For the mask models, the droplet's velocity can be added to the model input by accommodating the change velocity or reduced diameter before and after various mask types. For environmental models, the horizontal velocity, humidity, and wind velocity of the falling droplets can be added to the model by adding the training data according to the desired variables.

The proposed methods have potential functions as the fast prediction, interconnecting one research to another, gaining insight into unknown phenomena by working together with the numeric model. The model can duplicate the experimental or numerical data pattern; hence fast prediction of output or dependent variable with different input values or independent variables is possible without redo the simulation or experiment. Therefore, when predicting a situation or variable at a particular condition, the prediction can be carried in a short time. The model can also be employed to integrate one research to another, as demonstrated in the current paper, where the knowledge of two different research is integrated to predict the droplet flow from outlet mouth to the environment at various mask type conditions. Furthermore, the model can be employed to gain insight into an unknown phenomenon, mainly when reinforcement learning can be applied in the future.

\section{Conclusions}

A systematic approach to predict the final state droplet diameter and horizontal distance coverage is proposed. The approach can be divided into three parts, which are droplet generator, mask model, and environmental models. The droplet generator 
is developed based on measured data from coughing activities with an open mouth. The masked model is built based on an FFNN model trained by an ELM method. The environmental model has two models, predicting the final state droplet diameter and horizontal distance coverage. Both systems are modeled by FFNN trained by an ELM algorithm. The evaluation has shown promising results. The accuracy of the three machine learning models is relatively high, which R-value is more than 0.9900 . The evaporation condition can also be predicted successfully despite the condition not being included in the training range. The model can also predict the penetration time and has been validated with the previous work.

In summary, the model's application can be a fast evaluation of a prototype and consideration for the social distancing with various possible conditions. The model can also combine seemingly different scope studies while waiting for a valid experimental study or waiting for numerical solution solutions. The model needs to be further improved in the future, mostly to cover more data or cases such as more human activities and environmental conditions.

Author Contributions: Conceptualization, I.B. and S.B.W.; methodology, I.B. and J.T.P.; software, I.B. and J.T.P.; validation, I.B., A.F.M. and M.S.; formal analysis, I.B. and R.M.S.P.; investigation, C.A.P.; resources, N.N. and I.B.; data curation, N.N. and I.B.; writing-original draft preparation, I.B.; writing-review and editing, S.B.W., R.M.S.P. and A.F.M.; visualization, I.B.; supervision, I.B. and S.B.W.; project administration, C.A.P.; funding acquisition, I.B. All authors have read and agreed to the published version of the manuscript.

Funding: This research was funded by Vocational College, Universitas Gadjah Mada, through Hibah Penelitian Pemandatan with contract no. 227/UN1.SV/K/2020.

Conflicts of Interest: The authors declare no conflict of interest.

\section{References}

1. Duguid, J.P. The size and the duration of air-carriage of respiratory droplets and droplet-nuclei. Epidemiol. Infect. 1946, 44, 471-479. [CrossRef]

2. Kumar, V.; Nallamothu, S.; Shrivastava, S.; Jadeja, H.; Nakod, P.; Andrade, P.; Doshi, P.; Kumaraswamy, G. On the utility of cloth facemasks for controlling ejecta during respiratory events. arXiv 2020, arXiv:2005.03444.

3. Verma, S.; Dhanak, M.; Frankenfield, J. Visualizing droplet dispersal for face shields and masks with exhalation valves. Phys. Fluids 2020, 32, 091701. [CrossRef] [PubMed]

4. Johnson, G.R.; Morawska, L. The Mechanism of Breath Aerosol Formation. J. Aerosol Med. Pulm. Drug Deliv. 2009, 22, 229-237. [CrossRef]

5. Lai, A.C.K.; Cheng, Y.C. Study of expiratory droplet dispersion and transport using a new Eulerian modeling approach. Atmos. Environ. 2007, 41, 7473-7484. [CrossRef]

6. Vuorinen, V.; Aarnio, M.; Alava, M.; Alopaeus, V.; Atanasova, N.; Auvinen, M.; Balasubramanian, N.; Bordbar, H.; Erästö, P.; Grande, R.; et al. Modelling aerosol transport and virus exposure with numerical simulations in relation to SARS-CoV-2 transmission by inhalation indoors. Saf. Sci. 2020, 130, 104866. [CrossRef]

7. Shafaghi, A.H.; Talabazar, F.R.; Koşar, A.; Ghorbani, M. On the effect of the respiratory droplet generation condition on COVID-19 transmission. Fluids 2020, 5, 113. [CrossRef]

8. Redrow, J.; Mao, S.; Celik, I.; Posada, J.A.; Feng, Z.G. Modeling the evaporation and dispersion of airborne sputum droplets expelled from a human cough. Build. Environ. 2011, 46, 2042-2051. [CrossRef]

9. Aliabadi, A.A.; Rogak, S.N.; Green, S.I.; Bartlett, K.H. CFD simulation of human coughs and sneezes: A study in droplet dispersion, heat, and mass transfer. Asme Int. Mech. Eng. Congr. Expo. Proc. (IMECE) 2010, 7, 1051-1060. [CrossRef]

10. Li, X.; Shang, Y.; Yan, Y.; Yang, L.; Tu, J. Modelling of evaporation of cough droplets in inhomogeneous humidity fields using the multi-component Eulerian-Lagrangian approach. Build. Environ. 2018, 128, 68-76. [CrossRef] [PubMed]

11. Yang, S.; Lee, G.W.M.; Chen, C.M.; Wu, C.C.; Yu, K.P. The Size and Concentration of Droplets Generated by Coughing in Human Subjects. J. Aerosol Med. 2007, 20, 484-494. [CrossRef]

12. Busco, G.; Yang, S.R.; Seo, J.; Hassan, Y.A. Sneezing and asymptomatic virus transmission. Phys. Fluids 2020, 32, 073309. [CrossRef]

13. Dbouk, T.; Drikakis, D. On coughing and airborne droplet transmission to humans. Phys. Fluids 2020, 32, 053310. [CrossRef] [PubMed]

14. Shen, C.; Gao, N.; Wang, T. CFD study on the transmission of indoor pollutants under personalized ventilation. Build. Environ. 2013, 63, 69-78. [CrossRef] 
15. Li, Y.; Leung, G.M.; Tang, J.W.; Yang, X.; Chao, C.Y.; Lin, J.Z.; Lu, J.W.; Nielsen, P.V.; Niu, J.; Qian, H.; et al. Role of ventilation in airborne transmission of infectious agents in the built environment: A multidisciplinary systematic review. Indoor Air 2007, 17, 2-18. [CrossRef] [PubMed]

16. He, Q.; Niu, J.; Gao, N.; Zhu, T.; Wu, J. CFD study of exhaled droplet transmission between occupants under different ventilation strategies in a typical office room. Build. Environ. 2011, 46, 397-408. [CrossRef]

17. Forster, W.A.; Kimberley, M.O.; Zabkiewicz, J.A. A universal spray droplet adhesion model. Trans. Am. Soc. Agric. Eng. 2005, 48, 1321-1330. [CrossRef]

18. Sellens, R.W.; Brzustowski, T.A. A simplified prediction of droplet velocity distributions in a spray. Combust. Flame 1986, 65, 273-279. [CrossRef]

19. Arun, S.; Rakesh, P. Computational Evaluation of Spray Characteristics in Pressure Swirl Atomizers. Int. J. Sci. Eng. Res. 2015, 5, 804-809.

20. Dinc, M.; Gray, D.D.; Hillen, N.L.; Taylor, S.; Kuhlman, J.M.; Wv, M. Spray Simulations to Support the Development of a Monte Carlo-Based Spray Cooling Model. In Proceedings of the 43rd AIAA Fluid Dynamics Conference, New Orleans, Louisiana, 25-28 June 2012; pp. 1-24.

21. Chen, Q.; Xu, W. A zero-equation turbulence model for indoor airflow simulation. Energy Build. 1998, 28, 137-144. [CrossRef]

22. Gupta, J.K.; Lin, C.H.; Chen, Q. Flow dynamics and characterization of a cough. Indoor Air 2009, 19, 517-525. [CrossRef]

23. Cummins, C.P.; Ajayi, O.J.; Mehendale, F.V.; Gabl, R.; Viola, I.M. The dispersion of spherical droplets in source-sink flows and their relevance to the COVID-19 pandemic. Phys. Fluids 2020, 32, 083302. [CrossRef]

24. Bahiuddin, I.; Mazlan, S.A.; Shapiai, M.I.; Imaduddin, F.; Ubaidillah Choi, S.B. A new platform for the prediction of fielddependent yield stress and plastic viscosity of magnetorheological fluids using particle swarm optimization. Appl. Soft Comput. 2019, 76, 615-628. [CrossRef]

25. Huang GBin Wang, D.H.; Lan, Y. Extreme learning machines: A survey. Int. J. Mach. Learn. Cybern. 2011, 2, 107-122. [CrossRef]

26. Bahiuddin, I.; Imaduddin, F.; Mazlan, S.A.; Ariff, M.H.; Mohmad, K.B.; Choi, S.B. Accurate and fast estimation for field-dependent nonlinear damping force of meandering valve-based magnetorheological damper using extreme learning machine method. Sens. Actuators A Phys. 2021, 318, 112479. [CrossRef]

27. Cheng, G.J.; Cai, L.; Pan, H.X. Comparison of Extreme Learning Machine with Support Vector Regression for Reservoir Permeability Prediction. In Proceedings of the 2009 International Conference on Computational Intelligence and Security, Beijing, China, 11-14 December 2009; Volume 2. [CrossRef]

28. Neumann, K. Reliability of Extreme Learning Machines. Ph.D. Thesis, Bielefeld University, Bielefeld, Germany, 2014.

29. Feng, Y.; Marchal, T.; Sperry, T.; Yi, H. Influence of wind and relative humidity on the social distancing effectiveness to prevent COVID-19 airborne transmission: A numerical study. J. Aerosol Sci. 2020, 147, 105585. [CrossRef]

30. Bahiuddin, I.; Wahab, N.A.; Shapiai, M.I.; Mazlan, S.A.; Mohamad, N.; Imaduddin, F.; Ubaidillah. Prediction of field-dependent rheological properties of magnetorheological grease using extreme learning machine method. J. Intell. Mater. Syst. Struct. 2019, 30, 1727-1742. [CrossRef]

31. Bahiuddin, I.; Mazlan, S.A.; Shapiai, M.I.; Choi, S.B.; Imaduddin, F.; Rahman, M.A.; Ariff, M.H. A new constitutive model of a magneto-rheological fluid actuator using an extreme learning machine method. Sens. Actuators A Phys. 2018, 281, $209-221$. [CrossRef]

32. Chen, Y.; Wu, W. Mapping mineral prospectivity using an extreme learning machine regression. Ore Geol. Rev. 2017, 80, 200-213. [CrossRef]

33. Bahiuddin, I.; Mazlan, S.A.; Shapiai, M.I.; Imaduddin, F.; Ubaidilla. Study of extreme learning machine activation functions for magnetorheological fluid modelling in medical devices application. In Proceedings of the 2017 International Conference on Robotics, Automation and Sciences (ICORAS), Melaka, Malaysia, 27-29 November 2017. [CrossRef]

34. Zohdi, T.I. Modeling and simulation of the infection zone from a cough. Comput. Mech. 2020, 66, 1025-1034. [CrossRef]

35. Dbouk, T.; Drikakis, D. On respiratory droplets and face masks. Phys. Fluids 2020, 32, 063303. [CrossRef] [PubMed]

36. Bi, R. A Numerical Investigation of Human Cough Jet Development and Droplet Dispersion. Master's Thesis, The University of Western Ontario, London, ON, Canada, 2018.

37. Xie Li, Y.; Chwang, A.T.Y.; Ho, P.L.; Seto, W.H. How far droplets can move in indoor environments. Indoor Air 2007, 17, 211-2256. [CrossRef] [PubMed] 